\title{
XL.
}

\section{Beitrag zur Lehre von dem Adamantinom.}

\author{
Von \\ Dr. M. Matsuoka, \\ früherem Assistenten an der chirurgischen Klinik der Lniversitat Tokyo.
}

(Mit 4 Abbildungen.)

Vor drei Jahren exstirpierte ich einen Unterkiefertumor von einem 25jährigen Patienten in der unter meiner Leitung stehenden chirurgischen Abteilung der medicinischen Academie Saiseigakusha Tokyo. Das Interesse meines Falles, von den klinisehen Eigentümliehkeiten abgesehen, liegt in der histologisch charakteristischen Struktur der Geschwulst, über die bis jetzt nicht viel berichtet worden ist. Das nötigte mich, meinen Fall unseren Fachkollegen bekannt zu machen.

Vor fünf Jahren bemerkte der Patient eine diffuse, leicht auftreibende Anschwellung in der mittleren Partie des linken Cnterkieferkörpers ohne subjektive Beschwerden. Anfangs blieb die Größe der Anschwellung stationär; im Laufe des Jetzten Jahres begann die Anschwellung sich zn vergrößern, und erst in den Ietzten 3 Monaten wuchs diese ziemlich rasch an. Bei der Untersuchung bemerkt man eine circumseripte Anschwellung in dem linken Unterkieferkörper. Die Weichteile des Gesichts an der angeschwollenen Stelle sind nach anßen aufgetrieben nnd gespannt; es findet sich keine Hyperämie und kein Ödem. Die den Tumor bedeckende Gingiva sind stark nach außen und imnen aufgehoben; in einigen Stellen liegen die Geschwïre auf tief verlaufenden, eitrig secernierenden Fistelgängen. Die Zähne der betreffenden linken Unterkieferhälfte hinten rom zweiten Molarzahn vorn bis zum Eckzahn gingen total verloren; die ganze Unterkieferhälfte der linken Seite ist spindelförmig angeschwollen. Ihre äulere und innere Wand ist papierdünn; bei der Palpation fühlt man hier und da deutliches Pergamentknistern. An einigen Stellen ist die dünnwandige Knochenschale dureh feste Tumormasse perforiert; an anderen finden sich periostitische Knochenwucherungen, die höckerige Oberfläche erzengen. Der Tumor, von elastischweicher Konsistenz, ist von einer festen Bindegewebskapsel vollständig umschlossen, mit Ausnahme von einigen Fistelgängen. Der Tumor ist fast hühnereigrob. $\mathrm{Er}$ zeigt nirgends Drüsenmetastase. Die Operation wurde unter der Chloroformnarkose ausgeführt. Nach der Spaltung eventuell der Ablösung der den Tumor bedeckenden Weichteile wurde ein großes Stinck 
von der Knochenschale auf der äuBeren Seito des linken Unterkieferknochens herausgemeilielt. Die Kapsel der Geschwulst zeigte feste Verwachsung mit der inneren Fläche der Knochenschale und war ziemlich schwer von dieser zu trennen. Mittelst des scharfen Raspatoriums löste ich die Kapsel des Tumors von der Knochenschale ab, dann wurde die Geschwulst in toto herausgenommen. Nach exakter Blutstillung wurde eine plastische Operation mit dem gestielten Knochenperiostlappen ausgeführt, um den zu großen Defekt zu ersetzen. Die Weichteile wurden durch aseptische Seidenfäden zusammengenäht. Nach 5 Wochen war die Heilung tadellos.

Blutgefäße. Rete Malpighi. Zahnleiste.

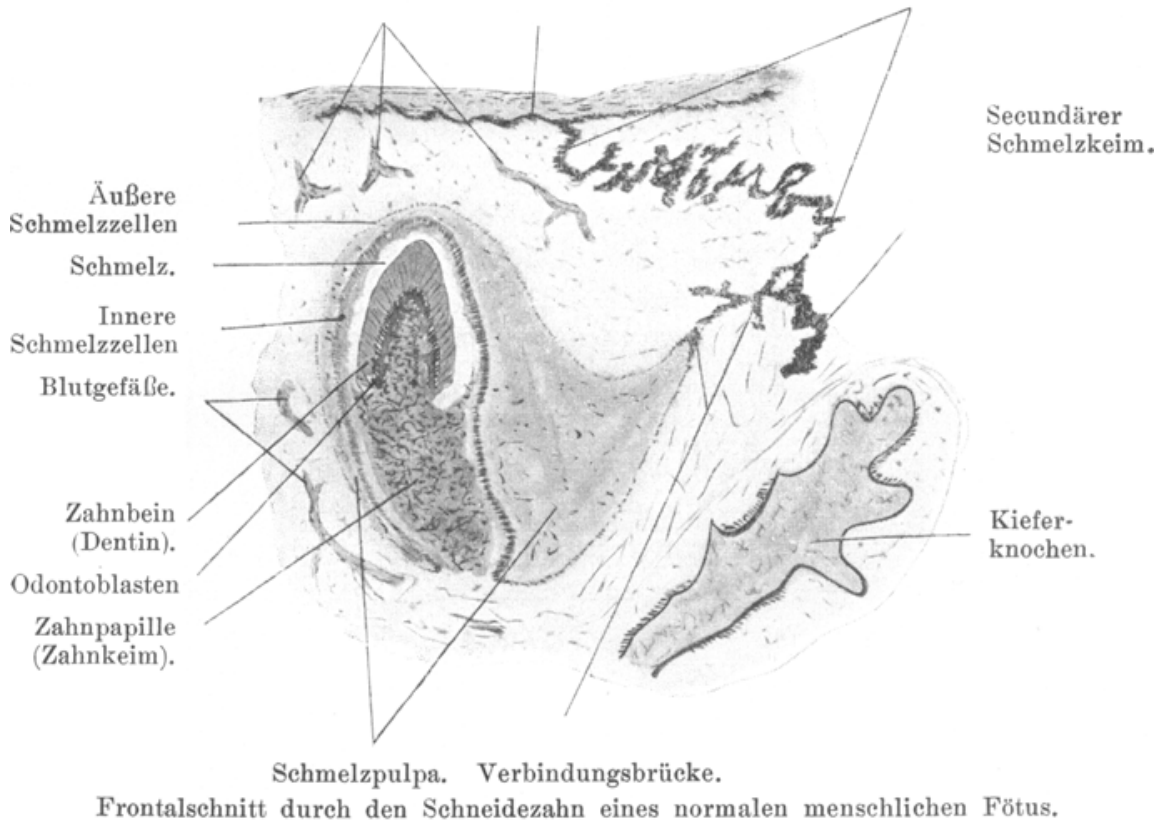

Fig. 1.

Makroskopisch zeigt die Tumormasse einen ondeutlichen lappigen Bau von weicher Konsistenz mit derber, fibröser Kapsel umschlossen. Zum Zweeke der mikroskopischen Untersuchung fixierte ich die Gowebsstückchen vom peripheren und zentralon Teile dieser Geschwulst in 10 proz. Formalinlösung und auch in $\mathrm{Z}$ enkerscher Flüssigkeit. Auswässerung, Alkoholnachhärtung und Paraffineinbettung gesehahen wie gewöhnlich. Zur Färbung wurden Muchämatein, S-Fuchsinpikrinsäure, Eisenhämatoxylin nach Heidenhain, 2 proz. Osmiumsänrelösung, 3 proz. Chromsäurelösung und polychromes Methylenblau verwendet. Mikroskopisch zeigt das Geschwulstparenchym einen drüsenartigen Bau mit dem sehr lockeren zellenreichen Bindegewebsstroma. Die Geschwulstkapsel ist nicht sehr dick und besteht aus parallel verlaufenden Bindegewebsfasern mit einer geringen Zahl von Blutgefäljen versehen. Bei der Färbung in Resorcin-Fuchsin- oder Orceïn- 
lösung kommen keine elastischen Fasern in Kapselgewebo zum Vorschein. $Z$ wischen den Bindegewelsfasern sind die den Sehnenzellen ähnlichen Elemente parallel eingelagert; diese sind bald sternförmig, bald spindelförmig, bald polymorph mit schmalen, nach beiden Enden verlängerten feineren, oft korkzieherartig gebogenen Ausläufern versehen. Iie auf obenerwähnte Weise gebildete, sehnig aussehende Kapsel ist von dem eigentlichen Geschwulstparenchym ziemlich seharf konturiert. Das letztere besteht ans zierlichen, fein verästelten, soliden Epithelzügen und zellenreichem Bindegewebsstroma. Auf der Schnittfläche des peripheren Geschwulstteiles sind die Epithelzapfon klein and vielfach verästelt mit sehr zeilenreichem Stroma. Nach dem zentralen Teile des Geschwulstparenchyms zu vermehrt der

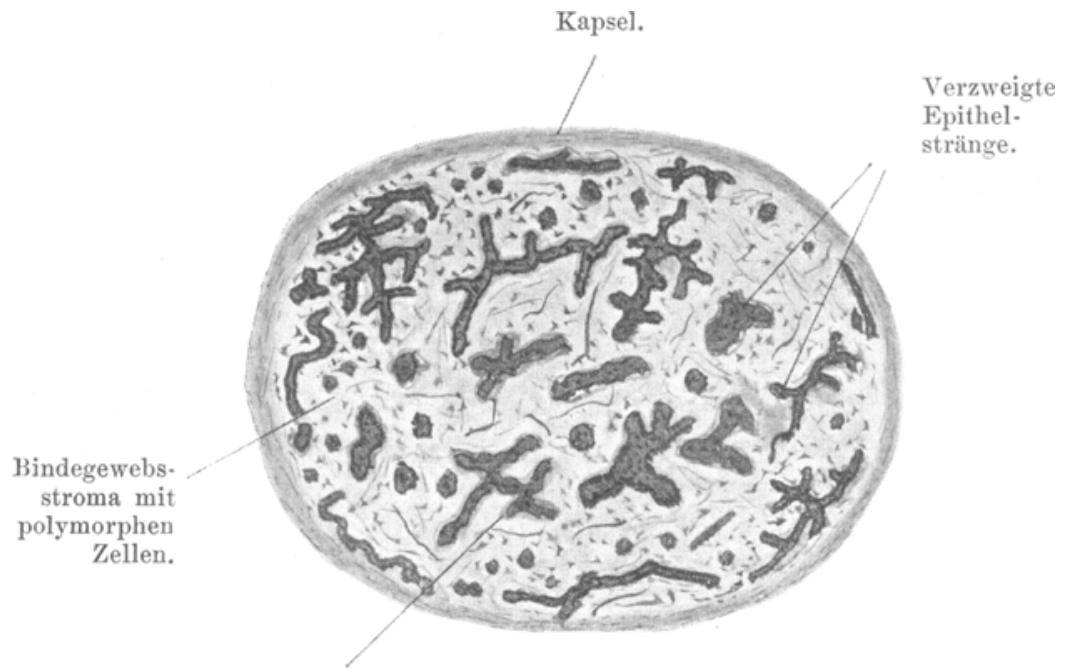

Zirkulär verlaufendes, homogenisiertes Bindegewebe.

Schema der Schnittfläche der ganzen Geschwulst.

Fig. 2.

Epithelzapfen sein Kaliber mit reduzierter Verästelung. Auf der Schnittfläche der Epithelschläuche zeigt sich, daß ihre periphere Lage von hochzylindrischen, dicht aneinandergereihten, bald ein-, bald mehrsehichtigen Epithelien besteht. Die Epithelkerne, deren Grenze gegen das Zellprotoplasma scharf conturiert ist, zeigen bald länglich ovale, bald an beiden Enden abgeruudete zylindrische Form. Der Kern enthält reichliche Menge ron Chromatinkörnern in ihrem Innern und liegt an dem gegen das Zentrum des Epithelschlanches gerichteten 'Teile der Zelle. Der periphere, kernlose, dem Bindegewebsstroma zugekehrte 'Teil des Zylinderepithels zeigt sich bald homogen, bald leicht granueiert; or bildet einen hellen Protoplasmasaum dureh Aneinanderreibung der kernlosen Teile der Epithelien. Im Zellleibe der Zylinderzellen befinden sich bald rundliche, bald ovale, bald polymorphe helle Lüeken mit dem Sekretstropfen ähnlichen Aussehen. Das Zentram des Epithelschlauches ist- mit großen polygonalen, meistens geflügelten Bindege. 
webszellen rersehen, ihr Kern zeigt eine bald rundliche, bald ovale Form und ist sehr chromatinreich. Jje Kerngrenze gegen das Zellprotoplasma ist ziemlich deutlich conturiert. Die feineren Ausläufer der einander benachbarten Epithelien kreazen sich netzartig. Zwischen diesen bemerkt man verschiedenartig gestaltete helle Iuicken. Manchual confluiert das Protoplasma einer Zelle mit dem der benachbarten und bildet eine gröbere Protoplasmamasse, während der Kern unverändert bleibt. Bei der Muchämateinfärbung ist die Mneinreaction sowohl in dem Zelleib, als auch in der metamorphosierten Protoplasmamasse negativ. In der peripheren Lage des relativ jüngeren, kleineren Epithelzapfens bemerkt man sehr zierliche,

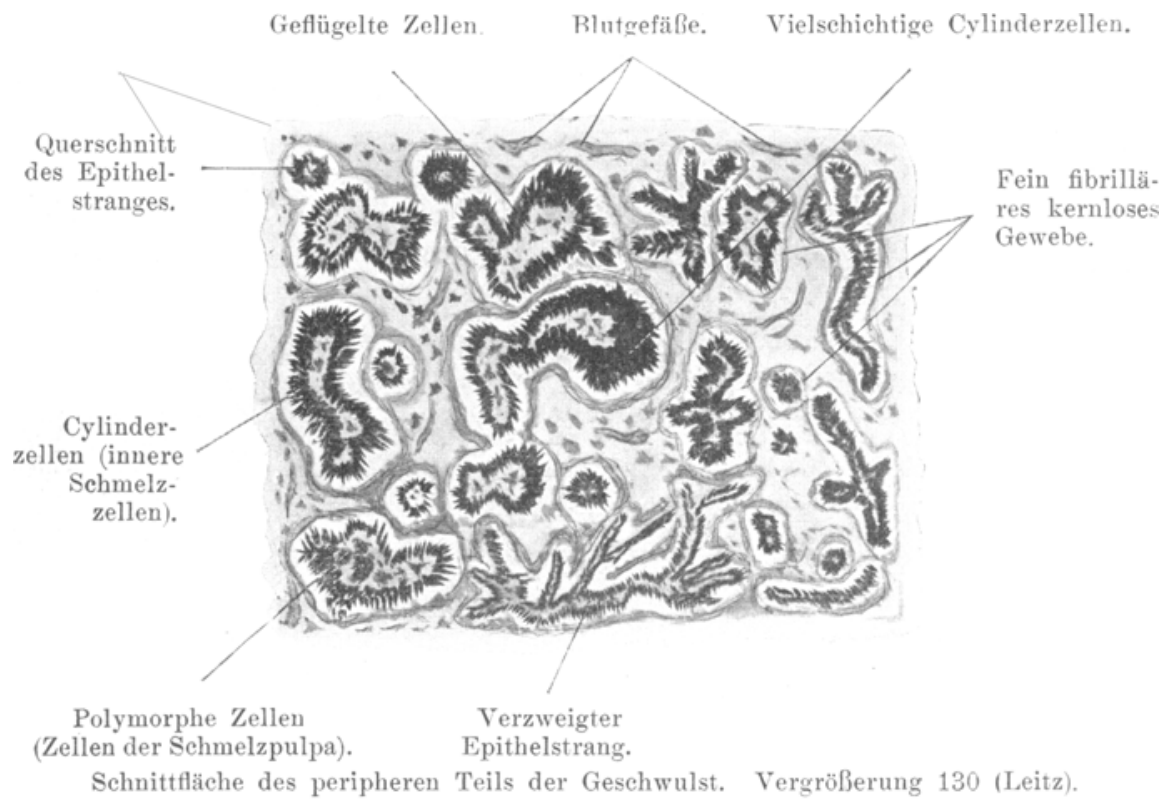

Fig. 3.

schlanke, dicht stohende, bald ein- bald mehrschichtige, dureh Hämateïn oder Mnchämateïn đ̉eutlich gefärbte Epithelzelien mit länglichen, feinen schmalen Kernen. Der' zentrale Teil des letzterwähnten Epithelschlauches besteht aus dicht gedrängten, protoplasmaarmen, unregeImäßig gestalteten, kleineren Zellen mit großen, chromatiureichen Kernen; hier fehlt dem Zellleib die helle Lücke, die in dem zentralliegenden des etwas älteren Fithelzapfens reichlich Forhanden ist. Die oben erwähnten Bilder der Fipithelgebilde sieht man besonders schön und deutlich bei der Färbung in Muchämateïnlösung. Jeder Epithelzapfen ist in dem kernarmen, bald zirkulär verlaufenden, bald büschelförinig g'ebundenen, feinen Fibrillen eingreschlossen. Dieses fibrilläre Gewebe entspricht ganz der Iembrana propria eines Drisenalveolus und ist nach v. Gieson besonders deutlich gefärbt. I) as Stroma besteht aus verschieden gestalteten, reichlichen Bindegewobszellen und netzartig gekreuzten feinen Fasern in sehr lockerer Terbindung. Die Zellen sind bald sternförmig, bald 
spindelförmig, hald polymorph mit feinen, langen Ausläufern versehen; der Kern zeigt sich polymorph und ist chromatinreich. Das Stroma ist im ganzen selr gefäßarm.

Es handelte sich um einen langsam wachsenden, abgekapselten, hühnereigroßen Tumor im Innern des linken Lnterkieferknochens bei einem 25 jährigen Yanne. Nach des Exstirpation des Tumors war die Wunde per primam geheilt; zwei Jahre lang bekam der Patient kein Reci-

\section{Blutgefäße. Bindegewebsfasern.}

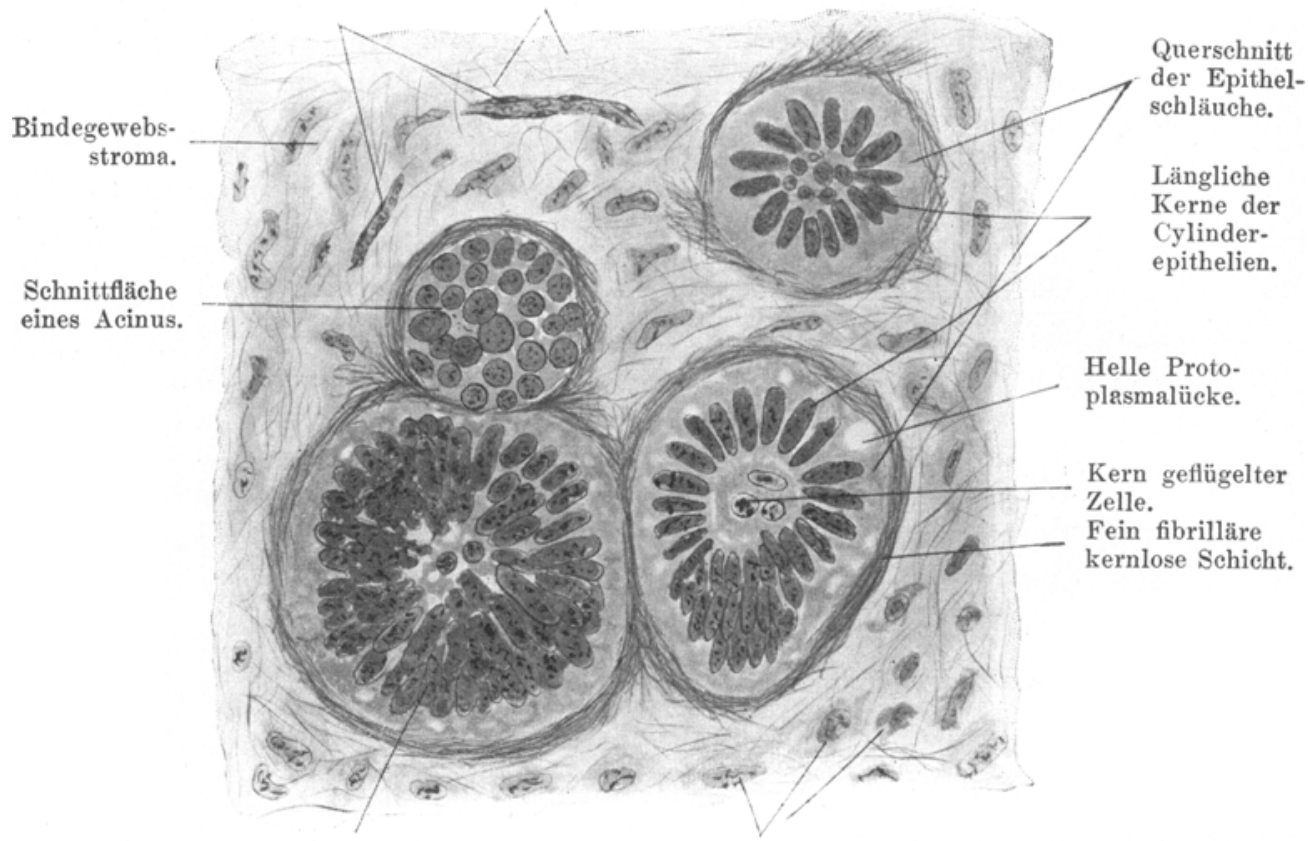

Mehrschichtige Cylinderzellen. Bindegewebszellen.

Schnittfläche des peripheren Teils der Geschwulst. Vergrößerung 600 (Leitz).

Fig. 4.

div. Der Tumor ist klinisch und makroskopisch gntartig. Histologisch ergibt sich, daß die drüsenartig gebauten Epithelgebilde in einem lockeren, zellenreichen Bindegewebe eingelagert sind. Bei flüchtiger Betrachtung ähnelt dieser Tumor einem Drüsenkrebs. Der Hauptunterschied ist, dab der erstere klinisch von gutartiger Natur ist und histologisch keinen atypischen Bau mit selbständigem Wachstum der Geschwulstelemente zeigt. Die Struktur des Epithelzapfens trscheint teils acinös, teils tubulös. Die äußere Schicht des tubulösen Zapfens besteht aus hochzylindrischen Zellen, deren kernloser Teil dicht an 
der Basalmembran liegt. Diesen letzterwähnten Befund bemerken wir niemals bei Karzinom and Adenom. Bei beiden letzten ist der kernlose Teil des Zylinderepithels gegen das Lumen zu gelegen. Vergleichen wir den histologischen Befund meines Falls mit dem des Zahnschmelzes auf dieser Entwickelung des Embryonallebens, so ergibt sich, dab die Struktur der Episthelien voliständiganalogist. Die hochzylindrischen Zellen entsprechen den inneren Schmelzzellen, während im zentralen Teile des Episthelschlauches die verschieden gestalteten, mit flügelartigen Fortsätzen versehenen Zellen von der Form der Sehmelzpulpa liegen. Bei der Färbung in Osmiumsäure oder Chromsäurelösung konnte ich die Schmelztropfen im Zelleib des Zylinderepithels nicht finden, welche Spee im embryonalen Schmelzepithel nachgewiesen hat.

Die im vorliegrenden Falle beschriebenen Befunde sind bereits teils histologisch, teils klinisch beschrieben worden ron Massin, Ma lassez, $\mathrm{Kr}$ u se und vielen anderen. In allen diesen Fällen gibt die Geschwulst ein gut charakterisiertes Bild vom Bau des Schmelzorganes. In den meisten Fällen entwickelt sich diese Geschwulst in jüngeren Lebensjahren und zwar in der Zeit vom 6. bis 30. Jahre; jedoch hat man diesen Tumor sowohl beim Neugeborenen (Massin) als auch bei älteren Leuten, z. B. bei einem 48 Jährigen (Gerken) beobachtet.

Die langsame, ohne Schmerzen und anderweitige Beschwerden allmählich zunehmende Entwickelung des Tumors, der innerhalb des Kieferknochens sitzt mit ausgesprochener Neigung, die innere und äubere Fläche des Kieferknochens vorzutreiben, ist in meinem Fall dieselbe wie in vielen anderen. Der weitere Verlauf zeigt die Gutartigkeit dieser Geschwulst. Das veranlassende Moment der 'Tumorentwickelung ist unklar; man hat es einmal als Entzündung oder Trauma betrachtet ohne sicheren pathologisch-anatomischen Bewcis. In vielen Fällen ist dieser Tumor cystisch (Ha asler, Becker usw.), jedoch auch solid wie in meinem Falle. Bei der Vergleichung der ganzen Literatur findet man verschiedene Übergangsstufen zwischen soliden und multilokulären Formen.

$\mathrm{König} \mathrm{nannte} \mathrm{diese} \mathrm{letzteren} \mathrm{follikuläre} \mathrm{Cystoide.} \mathrm{Bei} \mathrm{nieinem}$ Falle besteht der Tumor ausschließlich aus' Epithelzapfen und Bindegewebsstroma, die Cyste ist nirgends zu finden; jedoch finde ich intra- und interzelluläre helle Lüicken an vielen Stellen. Bezüglich der Genese dieser Epithelgeschwulst sind die Ansichten geteilt. Es fragt sich nun, ob dieser Tumor aus Schmelzzellen oder aus Schlcimhautepithelien resp. Driisenzellen der Mundhöhle stammt. Kolaczek schrieb, daf die ausgekleideten Epithelien der Cyste im Kieferknochen 
von der Mundschleimhaut ausgehen. Büchtemann, Eve und H eath berichteten auch, dab das multilokuläre Cystom aus dem Zabnfleischepithel entstchen. Magitot unterstützt von zablreichen casuistischen Lntersuchungen, betrachtete die an der Cystenwand liegenden Epithelien als die abundanten zelligen Elemente des Schmelzorganes. Später bestätigte Fal ks on die Magi to tsche Anschauung. Aus der histologischen Untersuchung durch Falk so n ergibt sich, daß die Epithelien von Zylinder- und Sternform mit alveolärem Bau entweder von einem iberzähligen Schmelzorgan oder von einem abnorm versprengten Schmelzkeim ausgehen. Malassez berichtete, daß diese Geschwulst aus dem zwischen Zahnanlage und Mundschleimhat anliegenden Fpithel, dem sog. Debris paradentaires entsteht. Das sind die Zahnleiste oder die Verbindungsbrücke, die sich beide zwischen Zahnanlage und Mundepithel in der Entwickelungszeit des Schmelzorganes befinden. Massin fand zwei gestielte, derbe epitheliale Tumoren in dem Zahnfleisch des Oberkiefers bei einem neugeborenen Kinde weiblichen Geschlechts. Die mikroskopische Untersuchung ergab, dal die epithelialen Elemente der beiden Geschwülste denen vom Schmelzorgan ähneln. Was den histologischen Befund bei meinem Falle betrifft, besteht der zapfenförmige Bau aus zylinder-und sternförmigen Zellen, die den Epithelien des embryonalen Schmelzorganes analog sind; es hat nichts gemein mit dem Bilde des Mundepithels. Durch den letzterwähnten Befund wissen wir, dab der Tumor bei meinem Fall mit dem sog. Adamantinom völlig übereinstim mt. Dic Frage, ob das Schmelzepithel entweder von der Zahnleiste oder von der Verbindungsbrücke oder vom abnorm verlagerten Schmelzkeim ausgeht, ist bei meinem Fall schwer zu entscheiden.

\section{Literaturverzeichnis.}

1) Graf Spee, Über die ersten Forgänge der Ablagerungen des Zahnschmelzes. Anatomischer Anzeiger. 2. Jalurg. $188 \%$.

2) Yassin, Fin Fall von angeborenem Fpitheliom, entstanden aus dem Schmelzorgan. Virchows Archiv. 136. Bd, 1594.

3) Mallassez, Sur le rôle des débris épithéliaux paradentaires. Archives de Physiologie norm. et pathol. Serie III. Tome V.

4) Kruse, Ëber die Entwickelung eystischer Geschwülste im Lnterkiefer. Virchows Archiv. 124. I3d.

5) Gerken, Zul Frage über die proliferierenden Cysten der Kiefer und Highmorshöhle. (Zitiert nach Massin.)

6. Ha asler, Die Histogenese der Kiefergesch wülste. Langenbecks Archiv. 53. Bd.

7) Kolaczek, Ein crstisches $A$ denom des Lnterkiefers. Arehiv f. klin. Chir. 21. Bd.

S) Büchtemann, Cystom des Unterkiefers. Archiy f. klin. Chirurgie. 26. Bd.

9) Eve und Heath zitiert nach Albarran: Rev. de chir. 1898.

10) Magitot, Sur les tumeurs du périost dentaire. 1559. Gaz. des Hospit.

11) Falkson, Zur Kenntnis der Kiefereysten. Virchows Archiv. 76. Bd. 